magnetic waves, whether transverse or longitudinal, pulse-like or periodic. $\mathrm{He}$ stresses the key role that the pulse interpretation played in the transition between classical and quantum viewpoints. Once the wave view predominated, studies of the ionizing properties of the new radiation confronted physicists with what Wheaton calls the paradoxes of quantity and quality: how could any wave-like disturbance, whose energy spreads out spherically from a central target, ionize only a few of the atoms in the surrounding region; and how could secondary electrons so produced each have about as much energy as the primary electrons whose deceleration at the target produced the rays in the first place?

Physicists here faced the fundamental paradox which was to remain unresolved for two decades: a wave-like disturbance manifested properties most readily explained by the assumption that the disturbance consisted of particles! Attempts to dodge this paradox are considered by Wheaton, notably the triggering hypothesis (X-rays only trigger the release of energy stored within the atom) and Bragg's attempt to suppress the wave aspect of the paradox by asserting that $\mathrm{X}$-rays were nothing but a particle beam. Such attempts were definitively ruled out by the discovery in 1912 of X-ray diffraction by crystals.

Similarly paradoxical features of visible light had been elucidated since 1905 by Einstein who, by 1909 , was publicly calling for a theory of light which somehow united wave and corpuscular aspects. Wheaton suggests that "our story developed quite independently" of Einstein's "imaginative and prescient statistical treatment of light" (p.xvii). But recently discovered letters from Einstein to Sommerfeld (Physikalische Blätter 40, 29; 1984) show that Einstein was involved in the discussion, during 1909 and 1910 , between Stark and Sommerfeld over the interpretation of the observed angular asymmetry in X-ray intensity. Einstein stressed the paradoxical features, insisting (at a time when this was not clear to everyone) that light and X-rays differ "only quantitatively".

The little Wheaton has to say about Einstein's role in the quantum story includes one major inaccuracy. He insists that Einstein's special theory of relativity was "diametrically opposed to the aether ontology", and succeeded in "eliminating the aether altogether" (p.106). Einstein was at pains to stress (in one of the aforementioned letters to Sommerfeld, among other places) that relativity theory required no stand on such ontological issues. It forced relinquishment of a mechanically interpreted aether, but was quite compatible with a non-mechanical aether. Indeed, Einstein's earliest attempt to resolve the wave-particle paradox was a unified theory (of electrons and light quanta) based on such a non-mechanical aether field.

X-ray diffraction work, together with the inhibiting effects on research of the
First World War, put a temporary stop to most experimental studies of the particulate aspects of radiation (with the notable exception of Millikan's work on the photoelectric effect). The great successes of the Bohr theory from 1913 on in understanding atomic structure and identifying atomic energy levels posed most acutely the question of the photoelectric effect and its $\mathrm{X}$-ray and $\gamma$-ray analogues. Post-war work on these problems made it more and more difficult to avoid facing up to the waveparticle paradoxes, first glimpsed over a decade earlier. Maurice de Broglie's group in Paris, which included his younger brother Louis, played a prominent role in this research. Wheaton shows how this work formed a natural background out of which Louis's brilliant idea of transferring the wave-particle paradox from radiation to matter could emerge. With a brief indication of the stimulating effect of de Broglie's work on the ideas of Einstein and Schrodinger and the experiments of Davisson and Germer the book ends. Readers may want to consult Vol.1 of Mehra and Rechenberg's recent Historical Development of Quantum Theory (Springer-Verlag, 1983) for additional details on and somewhat different interpretations of de Broglie's work and various other topics treated by Wheaton.

Wheaton tries to weave into the book a running contrast between "the mechanistic bias of British physicists" leading to the "British proclivity to seek mechanical models" (pp.5-6) and "German mathematical physicists" who "were not deterred by an inability to represent physical concepts mechanically" (p.7). He himself notes that "Larmor in Britain held views similar to those of many Germans" (p.7) and we can at once add the names of Poynting, Campbell, Pearson and Eddington to the list of British crusaders against mechanism. On the other hand, prominent German theoretical physicists such as Voigt, Boltzmann and Helmholtz (and the adolescent Einstein in his first scientific essay!) expended considerable effort to construct mechanical models of the electromagnetic aether. Given that we are talking about a small number of physicists in each country, it is hard to see which names go to make up the rule and which constitute the exceptions. It would seem wiser at this time to try to trace out the growth and interrelationship of smaller units, such as schools centred around leading figures or centres of physics research, rather than attempt to make sweeping generalizations about national styles of scientific research. For example, how would Wheaton explain the fact that "Deutsche Physik", of which Lenard and Stark were to boast a decade after Wheaton's story ends, prided itself on just those characteristics he attributes to British proclivities?

John Stachel is Professor of Physics at Boston University, and Editor of the collected papers of Albert Einstein, the first volume of which is scheduled to appear next year.

\section{Putting the heavens to order}

\section{Owen Gingerich}

Ptolemy's Almagest.

Translated and annotated

by G.J. Toomer.

Duckworth, London/Springer-Verlag, New York: 1984. Pp.693. £55, \$64.

Cl.Audius Ptolemy's geocentric system has long since been dumped into the dustbin of discarded theories. Why, then, is anyone still concerned with his hoary treatise? It is because Ptolemy's Almagest, more than any other book, convinced people that the seemingly complex phenomena of the heavens could be represented by a simple

\section{IMAGE UNAVAILABLE FOR COPYRIGHT REASONS}

Star gazer - Ptolemy depicted on the Campanile del Duomo, Florence.

underlying mathematical description, one that afforded the possibility of continuing prediction of celestial events. Certainly this was a milestone in the development of science.

G.J. Toomer's new English edition of Ptolemy's classic treatise is more than just a fresh translation. It is a most intelligently arranged presentation with a running, critical commentary at the foot of nearly every page, and it is based on a new reading of both the Greek and Arabic manuscripts. Included are a score of supplementary diagrams that clarify (especially) Ptolemy's instruments and his complex latitude theory, a brief introductory section that clues in the reader with respect to the technical background that Ptolemy assumes (such as ancient calendarial systems), and three appendices that give examples of numerical problems and textual variants. What Toomer has produced is the best edition in any language, one that will remain the standard preferred text for years to come. Ptolemy's Almagest is clearly the most important volume for the history of ancient science since $O$. 
Neugebauer's A History of Ancient Mathematical Astronomy, published nine years ago by Springer-Verlag.

In many respects, Ptolemy's work is to applied mathematics as Euclid's Elements is to pure mathematics. The Almagest begins with instructions for computing the trigonometric chord function, and continues by applying these in a long series of proofs, constructions and specific derivations of numerical parameters from his observational material. Fortunately Toomer has not hesitated to use an anachronism, the equals sign (which did not come into common usage until the sixteenth century), and he has carefully arranged his equations with the equality at a fixed position in the middle of the page. He has also adopted a double degree sign, $\circ$, for the "demi-degrees" that Ptolemy uses in order to facilitate his clumsy trigonometric manipulations. This attention to detail has produced a new level of elegance for the work, and, in combination with highly specific running heads, makes Ptolemy accessible as never before.

Ptolemy's attempt to be the Euclid of applied mathematics was fraught with certain difficulties, because observational data are not quite as tidy as the abstractions of pure points, lines and spaces. It is thus not surprising that his pioneering effort harbours certain ambiguities and outright botches. Untamed nature is not so easily brought into a mathematical harness. In his preface, Toomer allows that Ptolemy has manipulated his material "in order to present an appearance of rigor in his theoretical treatment which he could never have found in his actual experience". Indeed, Ptolemy, like astronomers today, undoubtedly built his edifice on a great array of traditional techniques and data, rejecting, adjusting or incorporating them as he saw fit. To examine how these various elements blend together is both a fascinating and a worthwhile research endeavour.

Not everyone has been willing to investigate Ptolemy with such scholarly calmness. In his The Crime of Claudius Ptolemy, published in 1977, the Johns Hopkins geophysicist R.R. Newton maintains that Ptolemy's work is riddled with deceit and concludes that it would have been better for astronomy if the Almagest had never been written. Newton's conclusion is at worst so extreme as to be silly, and at best a debating point. The problems of the Almagest deserve critical and informed discussion, Toomer writes, but he adds that Newton's book "provides nothing of the kind, but rather tends to bring the whole topic into disrepute". Although Toomer chooses not to debate with Newton in the present forum (wisely, in my opinion), he scores numerous points in his careful examination of the text. Here are two examples.

- Ptolemy's tables for the mean motions of the planets do not come up to his own epoch except by an extra addition,

thereby bringing forth the charge in some quarters that these tables were pilfered from an earlier source. "Despite Ptolemy's clear statement here of his motivation [for the arrangement], it has been the subject of much fruitless debate", notes Toomer on p.140. "By the time he came to compose the Handy Tables, he had realized the inconvenience of this arrangement, and switched. ..".

- For some centuries there has been speculation that Ptolemy's extensive star catalogue, which occupies the better part of two of the 13 books comprising the Almagest, was taken over wholesale from his predecessor Hipparchus. There exists no direct evidence, however, for any systematic set of numerical positions compiled by the earlier astronomer. While Toomer does not argue for Ptolemy's originality in compiling the catalogue, he makes it clear that he believes any attempt to credit the positions to Hipparchus is unfounded. In commenting on the text

\section{Picture astronomy}

\section{Stephen P. Maran}

The New Astronomy.

By Nigel Henbest and Michael Marten. Cambridge University Press: 1983.

Pp.240. £12.50, \$24.95.

IN The New Astronomy, Nigel Henbest and Michael Marten celebrate the recent achievements made through digital image processing and (for some spectral regions) through the use of two-dimensional detectors. In computer-generated astrophysical images, colour may represent intensity or polarization of light, a physical parameter such as temperature or strength of magnetic field, or the rate of change of any such quantity with position in the sky. So not only are the pictures reproduced in this book of aesthetic appeal, they are pregnant with information.

Some of the most dramatic contrasts between separate views of the same object are provided by jovian imagery. Compared with a highly-detailed Voyager photograph of Jupiter, infrared scans show a seemingly more ordered planet, while radio and $\mathrm{X}$-ray images are so different from the usual picture that an uninitiated astronomer might well mistake the target. The X-ray Jupiter comprises two separate sources, but $6-\mathrm{cm}$ radio waves define a tripartite Jupiter, a central oval flanked by two smaller patches, orientated at right angles to the X-ray sources. Especially striking are correlated sequences depicting emission from the jovian magnetosphere: the pattern of $21-\mathrm{cm}$ wavelength continuum sources wobbles up and down as Jupiter spins, while in the corresponding radio polarization maps the dominant mode of circular polarization reverses as the planet presents first one magnetic pole towards leading up to the actual star list, he notes (p.330) that

all the evidence is in favour of the hypothesis that Hipparchus did not record stellar positions in latitude and longitude (except for a few special cases. . .). Otherwise it is impossible to explain why Ptolemy went through the cumbersome process of comparing declinations, instead of simply comparing latitudes observed by Hipparchus and himself.

Extensive though his notes are, Toomer does not speculate on the origins of the Ptolemaic planetary models, nor on their development in Ptolemy's later works. Nevertheless, this monumental volume now paves the way for further investigations of what, precisely, was going on in ancient applied mathematics, an analysis that should bring a fuller understanding of the roots of our own modern science.

Owen Gingerich is Professor of Astronomy and the History of Science at the HarvardSmithsonian Center for Astrophysics, Cambridge, Massachusetts.

\section{the Earth and then the other.}

The educational impact of the illustrations in The New Astronomy is typified by the colour-coded radial velocity maps of the Andromeda galaxy. From these it is clear which side of the galaxy is turning away from us and which side towards us; shades of red (as in redshift) denote recession velocities and approach velocities are represented by cooler green and blue, while off-colour patches signal velocity pattern disturbances that may correspond to density waves which maintain the spiral arms.

A few points, if addressed, should lead to an even better second edition of the book. The presentation of visible, ultraviolet and $\mathrm{X}$-ray solar images would be much improved if these were based on photographs made on the same date and thus referring to the Sun in the same condition. Consistency has gone too far in the captions for a set of ultraviolet, X-ray and gamma-ray sky maps. In each, the wavelength is expressed in nanometers, but this, together with deliberate avoidance of powers-of-ten notation, results in a description for one map (" $0.0000002-0.000$ $02 \mathrm{~nm}$ ") that neither novice nor expert can appreciate. Finally, some images need marker lines at the borders, or some judiciously applied arrows: few readers will identify Eta Carinae in the photograph of complex nebulosity from the information that the star is "at the lower-left end of the small banana-shaped dark cloud".

For the non-specialist, this is a fine guide to the spectacle of modern astrophysics. Professional astronomers will find the book, with its detailed list of picture sources, the right place to identify the best new illustrations for lectures and articles.

Stephen P. Maran is a Senior Staff Scientist in the Laboratory for Astronomy and Solar Physics at the NASA-Goddard Space Flight Center, Greenbelt, Maryland. 